\title{
FNDC3B promotes cell migration and tumor metastasis in hepatocellular carcinoma
}

\author{
Chin-Hui Lin ${ }^{1}$, Yao-Wen Lin ${ }^{1}$, Ying-Chun Chen ${ }^{1}$, Chen-Chung Liao ${ }^{2}$, Yuh-Shan Jou ${ }^{3}$, \\ Ming-Ta Hsu ${ }^{1}$, Chian-Feng Chen ${ }^{1}$ \\ ${ }^{1}$ VYM Genome Research Center, National Yang-Ming University, Taipei, Taiwan \\ ${ }^{2}$ Proteomics Research Center, National Yang-Ming University, Taipei, Taiwan \\ ${ }^{3}$ Institutes of Biomedical Sciences, Academia Sinica, Taipei, Taiwan
}

Correspondence to: Chian-Feng Chen, email: cfchen@ym.edu.tw

Keywords: FNDC3B, hepatocellular carcinoma, metastasis

Received: April 05, 2016 Accepted: June 13, $2016 \quad$ Published: July 01, 2016

\section{ABSTRACT}

Recurrence and metastasis are common in hepatocellular carcinoma (HCC) and correlate with poor prognosis. We investigated the role of fibronectin type III domain containing 3B (FNDC3B) in HCC metastasis. Overexpression of FNDC3B in HCC cell lines enhanced cell migration and invasion. On the other hand, knockdown of FNDC3B using short-hairpin RNA reduced tumor nodule formation in both intra- and extrahepatic metastasis. High levels of FNDC3B were observed in metastatic HCCs and correlated with poor patient survival and shorter recurrence time. Mutagenesis and LC-MS/MS analyses showed that FNDC3B promotes cell migration by cooperating with annexin A2 (ANXA2). Furthermore, FNDC3B and ANXA2 expression correlated negatively with patient survival. Our results indicate that FNDC3B behaves like an oncogene by promoting cell migration. This suggests FNDC3B could serve as a biomarker and therapeutic target for HCC metastasis.

\section{INTRODUCTION}

Hepatocellular carcinoma (HCC) is one of the most common and aggressive tumors worldwide [1,2]. In spite of therapeutic advancements that can extend the lifespan of patients [2], HCC is still the third leading cause of cancer-related deaths in the world [3]. HCC has a high frequency of recurrence after routine surgical treatment [4], and metastasis to the lungs, lymph nodes, bones, and adrenal glands is common $[5,6]$.

A genome-wide approach to identify common copy number alternation regions in the genome, targeting either amplifications or deletions, can reveal novel cancer genes. In a previous study, we screened for genomic aberrations in HCC cell lines and identified a $329 \mathrm{~kb}$ amplicon in 3 q26.3 containing only 1 gene, fibronectin type III domain containing $3 \mathrm{~B}$ ( $F N D C 3 B)$, which was upregulated in HCC tissues and cell lines [7]. In addition, the knockdown of $F N D C 3 B$ decreased anchorage independent growth (AIG) and tumor formation in xenograft models [7].

$F N D C 3 B$, also called FAD104 (factor for adipocyte differentiation 104), is a known regulator of adipocyte and osteoblast differentiation [8-10]. The fibronectin type III (FNIII) domains of the FNDC3B protein are involved in cell adhesion and growth signaling [11, 12]. Cell adhesion is important during tumorigenesis as it provides tumor cells with the necessary cell-to-cell contacts and cellmatrix interactions for cell signaling, proliferation, and migration [13]. In addition, the homozygous disruption of $F N D C 3 B$ causes rapid postnatal death in mice and has pronounced effects on the adhesion, spreading, and migration of mouse embryo fibroblasts [9]. Therefore, we hypothesized that $F N D C 3 B$ promotes cell migration and tumorigenesis in HCC. In this study, we investigated the role of FNDC 3B in HCC through in vitro and in vivo experiments to understand its mode of action.

\section{RESULTS}

FNDC $3 B$ promoted cell migration and invasion in $\mathrm{HCC}$ cell lines

FNDC3B was commonly expressed in HCC cell lines (Figure S1A). To study whether FNDC3B 
indeed promotes cell migration and invasion, full-length expression vector or shRNA for $F N D C 3 B$ was transfected into HCC cell lines. Knockdown or overexpression of FNDC3B does not significantly alter the cell growth rate (Figure S1B). As shown in Figure 1A, overexpression of $F N D C 3 B$ enhanced migration in most of the HCC cell lines test as compared with vector only. Interestingly, knocking down the expression of FNDC3B resulted in inhibition of cell migration and invasion (Figure 1B). In Mahlavu and Huh7 cells, migration and invasion decreased more than $30 \%$ when $F N D C 3 B$ was knocked down. This suggests that $F N D C 3 B$ promotes cell migration and invasion during tumorigenesis.

\section{FNDC3B knockdown in highly invasive Mahlavu cells reduced tumor nodule formation in vivo}

To validate our in vitro observations and investigate whether $F N D C 3 B$ could serve as therapeutic target for metastatic HCC, we established a stable cell line of Mahlavu cells with knockdown of FNDC3B. The stable knockdown cells or the control Mahlavu cells were mixed with matrigel and injected into nude mice by orthotopic intrahepatic or tail vein injection. Six weeks after injection, the mice were sacrificed and visible tumor nodules were observed on the surface of the liver and lungs. The mice receiving injections of $F N D C 3 B$ knockdown cells produced less than $60 \%$ tumor nodules compared with the vector control and original cells either by orthotopic intrahepatic (Figure 2A) or tail vein injection (Figure 2B) ( $p=0.0216$ and $p=0.0102$, respectively). Immunohistochemical analysis of tumor nodule sections indicated the tumor nodule expressed human FNDC3B (Figure $2 \mathrm{C}$ ). Consistent with our in vitro results, these results indicated that knockdown of $F N D C 3 B$ significantly inhibits metastasis in HCC.

\section{FNDC3B overexpression correlates positively with HCC metastasis and negatively with patient survival}

Both in vitro and in vivo studies suggested that the expression of $F N D C 3 B$ correlates positively with metastasis in HCC. To further confirm the role of $F N D C 3 B$ in tumor metastasis, we analyzed the expression of $F N D C 3 B$ in 15 metastatic and 44 primary $\mathrm{HCC}$ tissues by tissue array (Figure S2). Interestingly, FNDC $3 B$ was overexpressed in metastatic HCC tissues $(73.33 \%, 11 / 15)$ compared with primary HCCs $(45.45 \%, 20 / 44)(p=0.003)$ (Figure 3A-3B). To further validate our observations, we analyzed 242 HCC microarray data obtained from public databases. Kaplan-Meier analysis showed that upregulation of $F N D C 3 B$ correlated negatively with patient survival $(p<0.001)$ (Figure $3 \mathrm{C})$. The average survival time was 41.22 months for HCC patients with upregulated $F N D C 3 B$ and 52.37 months for patients without $F N D C 3 B$ upregulation. Patients with upregulated $F N D C 3 B$ presented a $64.65 \%$ recurrence rate and an average of 33.4 months recurrence time, while HCC patients without $F N D C 3 B$ upregulation had a recurrence rate of $51.1 \%$ and average recurrence time of 42.53 months $(p=0.016)$ (Figure 3D). Our survival analysis suggests the upregulation of $F N D C 3 B$ correlates positively with recurrence and negatively with patient survival.

\section{FNIII domains 1-4 of FNDC3B were essential for cell migration}

FNDC3B is composed of 9 FNIII domains and a transmembrane domain (Figure 4A). FNIII has been shown to serve as a scaffold for the generation of stable protein domains that bind to variable proteins $[14,15]$. To explore the role of FNIII domains in cell migration, we constructed partial FNIII deletion mutants of FNDC3B and analyzed their migration ability (Figure 4B-4C; Figure S3). The cellular distribution of flag-FNDC3B overlapped with the staining pattern of calnexin, a marker for the endoplasmic reticulum (ER), suggesting that FNDC3B localizes to the ER membrane (Figure 4B). The transmembrane domain deletion mutants redirected their cellular localization to the nucleus and lost the ability to promote migration (Figure 4B-4C). Therefore, cellular localization of FNDC3B on the ER membrane is important for the FNDC3B's migration-inducing activity. Of note, we found that the $\Delta 6 \sim 9$ mutants exhibited similar migration capabilities as the full-length construct, but the $\Delta 1 \sim 4$ mutants showed no difference compared with the vector only control (Figure 4C; Figure S3). These results suggest that the first four FNIII domains are responsible for FNDC3B's functions related to cell migration.

\section{FNDC3B cooperated with ANXA2 to promote cell migration}

Co-immunoprecipitation (co-IP) was performed to identify proteins interacting with wild type FNDC3B and $\Delta 1 \sim 4$ mutant. Proteins differentially binding to wild type and the mutant FNDC3B were analyzed by Liquid Chromatograph Tandem Mass Spectrometry (LC-MS/MS). One of the candidates, ANXA2, interacted with fulllength FNDC3B but not with the $\triangle 1 \sim 4$ mutant. $A N X A 2$ overexpression has been suggested to be an HCC marker $[16,17]$. The tyrosine-phosphorylated ANXA2 has also been shown to be involved in cell migration and transformation [18, 19]. In our immunoprecipitation experiments the full-length FNDC3B interacted with phosphorylated ANXA2 (Figured 5A). Cell migration assays indicated that $F N D C 3 B$ overexpression induced cell migration, which was reversed by knocking down $A N X A 2$ (Figure 5B). The same results were observed when $A N X A 2$ was overexpressed in FNDC $3 B$ knockdown cells (Figure 5B). In previous reports, phosphorylation of 


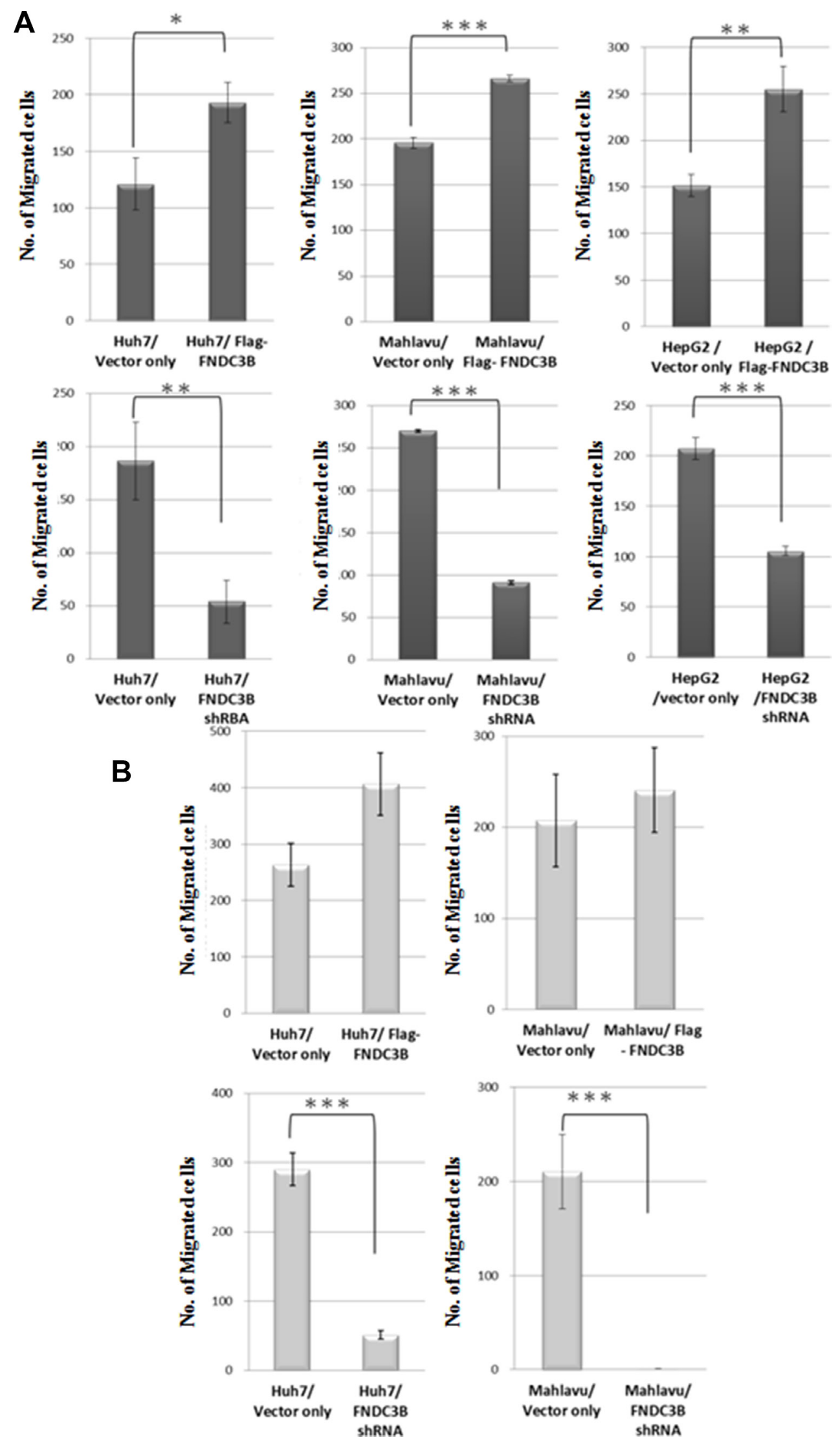

Figure 1: FNDC3B enhanced cell migration and invasion. (A) Migration assay for overexpressed or knockdown cells utilized a transwell system. (B) Cell invasion assays (similar to the migration assays) with collagen pre-coated inserts $\left(10 \mu \mathrm{g} / \mathrm{cm}^{2}\right)$. **and $\mathrm{d}^{* * *}$ represent $p<0.005$ and $p<0.0005$, respectively. 
ANXA2 has been shown to regulate Rho-mediated actin rearrangement [20-22]. In our immunofluorescence staining experiments, cells overexpressing FNDC3B presented more well-defined stress fibers along the cellular length (Figure 5C). In addition, Rho inhibitor (Y-27623) treatment reduced migration in cells overexpressing FNDC3B $(p<0.005)$ (Figure 5D). These observations suggest that FNDC3B cooperated with ANXA2 to promote cell migration by mediating actin rearrangement. Furthermore, we performed Kaplan-Meier analyses to measure the correlation between $F N D C 3 B$ or $A N X A 2$ expression and $\mathrm{HCC}$ patient survival. Interestingly, the upregulation of $A N X A 2$ did not alter patient survival when $F N D C 3 B$ was expressed at normal levels (52.15 months survival time compared to 52.36 when $A N X A 2$ was expressed at normal levels). However, the upregulation
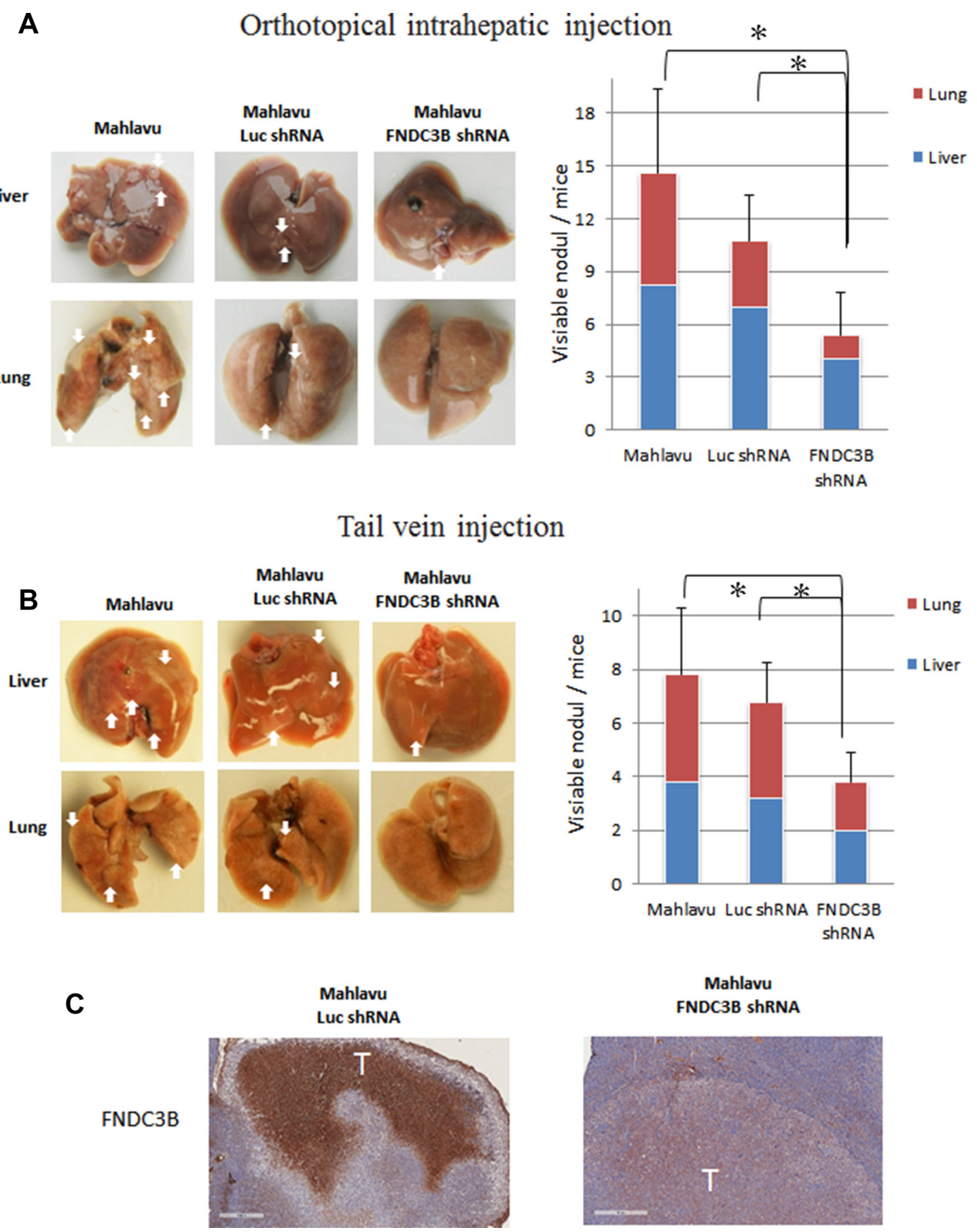

Figure 2: Knockdown of endogenous FNDC3B expression suppressed tumor metastasis in nude mice model. Metastatic nodules in nude mice by (A) orthotopical intrahepatic or (B) tail-vein injection of Mahlavu cells. The white arrows indicate metastatic tumors. The metastatic number in liver and lung was calculated in nude mice. *represents $p<0.05$. (C) Immunohistochemical staining of FNDC3B in liver tumor model section. Scale bar $=400 \mu \mathrm{m}$. 
of $A N X A 2$ decreased patient survival when $F N D C 3 B$ was also upregulated (37.78 months survival time compared to 46.45 months when $A N X A 2$ was expressed at normal levels, $p=0.003$ ) (Figure 5E).

\section{DISCUSSION}

In this study, we performed in vitro cell migration and invasion assays, in vivo metastasis assays, tissue array analyses, and survival analyses of patients to study the role of FNDC $3 B$ in HCC metastasis. Knocking down FNDC $3 B$ expression prevented intra- and extra-hepatic metastasis in our metastasis mouse model. Clinical data also indicated that the expression of $F N D C 3 B$ correlated positively with HCC metastasis and negatively with patient survival. Additionally, protein interaction, cell migration and survival analyses suggested that FNDC3B cooperated with ANXA2 to promote cell migration and invasion.

By analyzing microarray data from public domain databases, we previously showed that $F N D C 3 B$ was upregulated in HCC samples infected with $\mathrm{HBV}$ or $\mathrm{HCV}$ [7]. Here, our tissue array data showed that $F N D C 3 B$ was highly expressed in normal liver tissue infected with viral hepatitis $(45 \%, 9 / 20)$ (Figure S4). This observation suggests that the expression of $F N D C 3 B$ correlates with hepatic virus infection. Since hepatic virus infection is the major cause of $\mathrm{HCC}, F N D C 3 B$ would be important for the tumorigenesis of $\mathrm{HCC}$.

FNDC3B is mainly composed of FNIII domains. Individual FNIII repeats have a high degree of structural homology, despite displaying only $20-40 \%$ identity in their amino acid sequences. They are composed of $\sim 90$
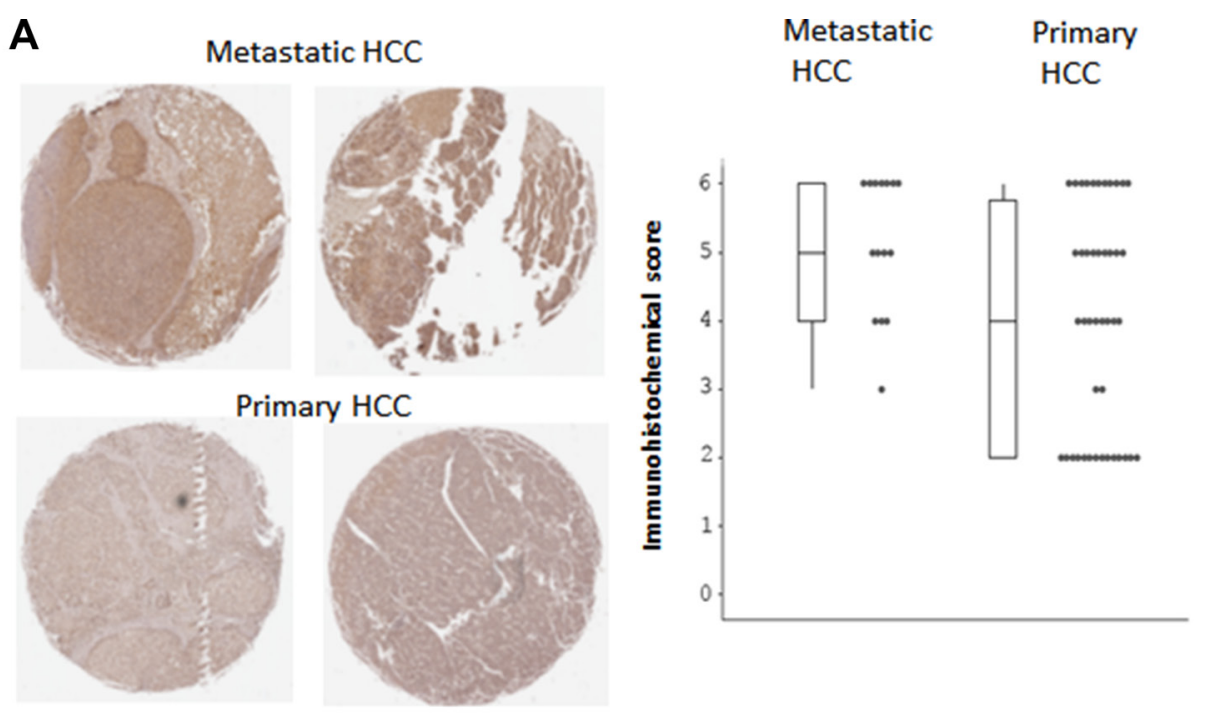

B

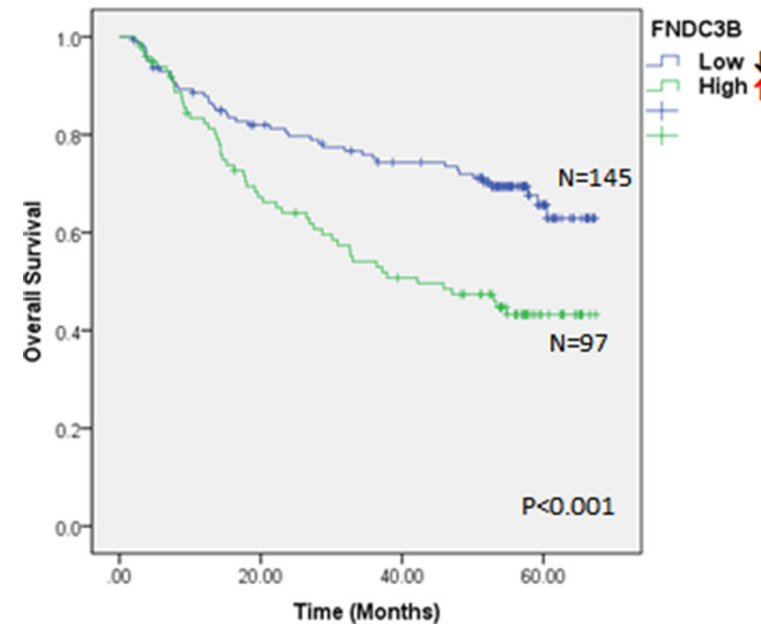

C

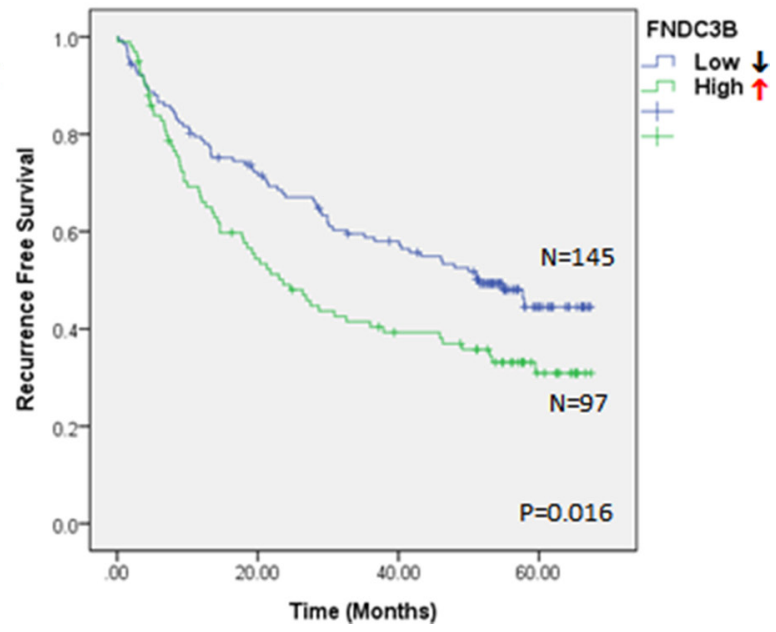

Figure 3: FNDC3B overexpression correlates with HCC metastasis and patient survival. (A) FNDC3B levels in tissue arrays detected by FNDC3B antibodies in metastatic and primary HCC. (B) Differential immunohistochemical (IHC) score of FNDC3B between metastatic and primary HCC. (C) Overall survival and (D) recurrence-free survival of HCC patients categorized accordingly to the expression of $F N D C 3 B$. $\uparrow$ and $\downarrow$ stand for high $(\mathrm{T} / \mathrm{N} \geq 1.5)$ and low $(\mathrm{T} / \mathrm{N}<1.5)$ expression groups, respectively. 
amino acids organized in 7 anti-parallel $\beta$ strands and are involved in cell adhesion and growth signaling [23]. In this study, we constructed domain deletion mutants to identify the FNDC3B domains that are involved in cell migration. Our results indicated that FNIII domains 1 4 are important for cell migration. Moreover, the transmembrane domain deletion mutant redirected the localization of FNDC3B in cells from the ER to the nucleus and FNDC3B lost its ability to promote cell migration. These results suggest that FNDC3B's localization to the ER membrane is essential for activating the cell migration signaling pathway.

ANXA2 is a calcium-dependent phospholipidbinding protein, which can cap filamentous actin to regulate membrane-membrane and membrane-cytoskeletal interactions [24]. ANXA2 has been implicated in many cellular functions such as exocytosis, endocytosis, vesicle transport, ion channel regulation, immune response, cellto-cell adhesions, and fibrinolysis [24, 25]. ANXA2 can be phosphorylated at Tyr23 by Src kinase [26] to regulate Rho mediated actin rearrangement and cell morphology alteration [20-22]. ANXA2 is aberrantly expressed in many types of cancer, including HCC [27-30]. In addition, tyrosine-phosphorylated ANXA2 is involved in cell

A

B

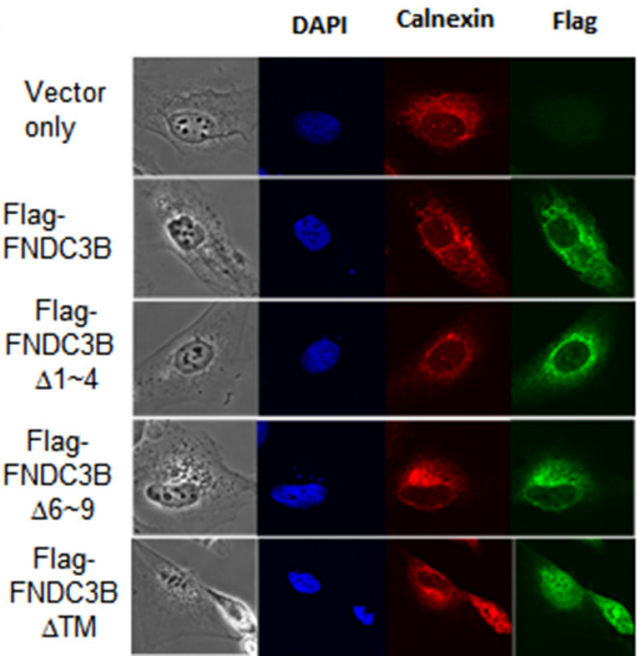

migration and malignant transformation [18]. We observed that FNDC3B interacts with phosphorylated ANXA2 through the first four FNIII domains, which are essential for cell migration. Immunofluorescence staining and Rho inhibitor (Y-27623) treatment suggested that FNDC3B might rearrange actin by cooperating with phosphorylated ANXA2. However, further studies are necessary to understand the underlying mechanism.

$F N D C 3 B$ is one of the most commonly upregulated genes in cancerous tissues, regardless of cancer type and tumor origin [31]. Here, we showed that $F N D C 3 B$ was overexpressed in the metastatic HCC tumors and that $F N D C 3 B$ expression correlated negatively with HCC patient survival. We also performed Kaplan-Meier survival analyses using microarray data (http:/www. kmplot.com) from thousands of breast and ovarian cancer patients. $P$ values $\left(p=2 \times 10^{-15}\right.$ for breast cancer and $p$ $=0.00057$ for ovarian cancer) indicated that FNDC3B expression also correlated negatively with patient survival in other cancers (Figure S5).

In a previous report, Zhang et al. found that miR-143 was highly expressed in a subset of metastatic HCC cases, positive for hepatitis B virus. Such study suggested that

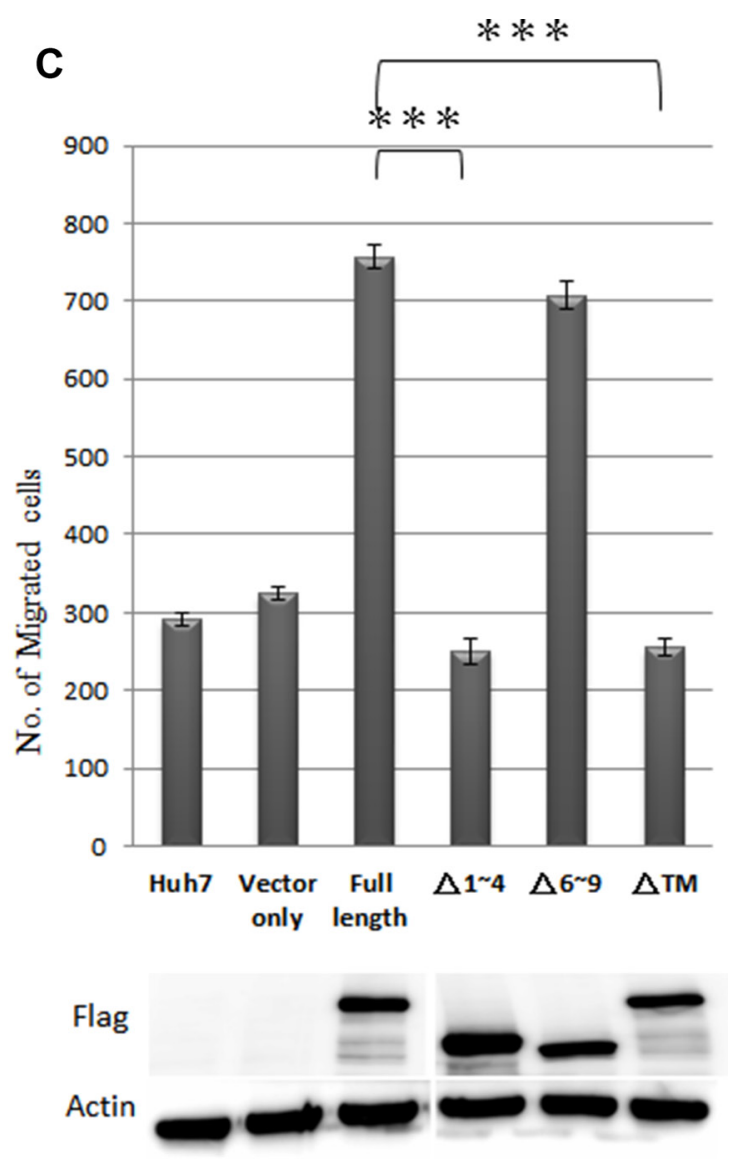

Figure 4: FNIII domains 1-4 were essential for FNDC3B to enhance cell migration and invasion. (A) The construction map of variant FNIII domain deletion mutants of FNDC3B. (B) Cellular localization of full-length $F N D C 3 B$ and variant domain deletion mutants assessed by immunofluorescence. Calnexin and Flag-FNDC3B visualized with TRITC-conjugated calnexin (red) and anti-Flag antibody (green), respectively. (C) Migration assays for FNIII domain deletion mutants performed using the transwell system. ***represent $p<0.0005$. 
miR-143 promoted metastasis by inhibiting the expression of $F N D C 3 B$ [32]. However, other evidence highlights $F N D C 3 B$ as oncogene. First, $F N D C 3 B$ is usually amplified [7, 33] and highly expressed in HCC and other cancers [7, 31, 34-36]. Cai et al. found that FNDC3B overexpression induces the epithelial-to-mesenchymal transition and activates several cancer pathways [33]. In addition, direct evidence from our knockdown and overexpression experiments indicates that $F N D C 3 B$ promotes cell migration and tumor metastasis in HCC.
A
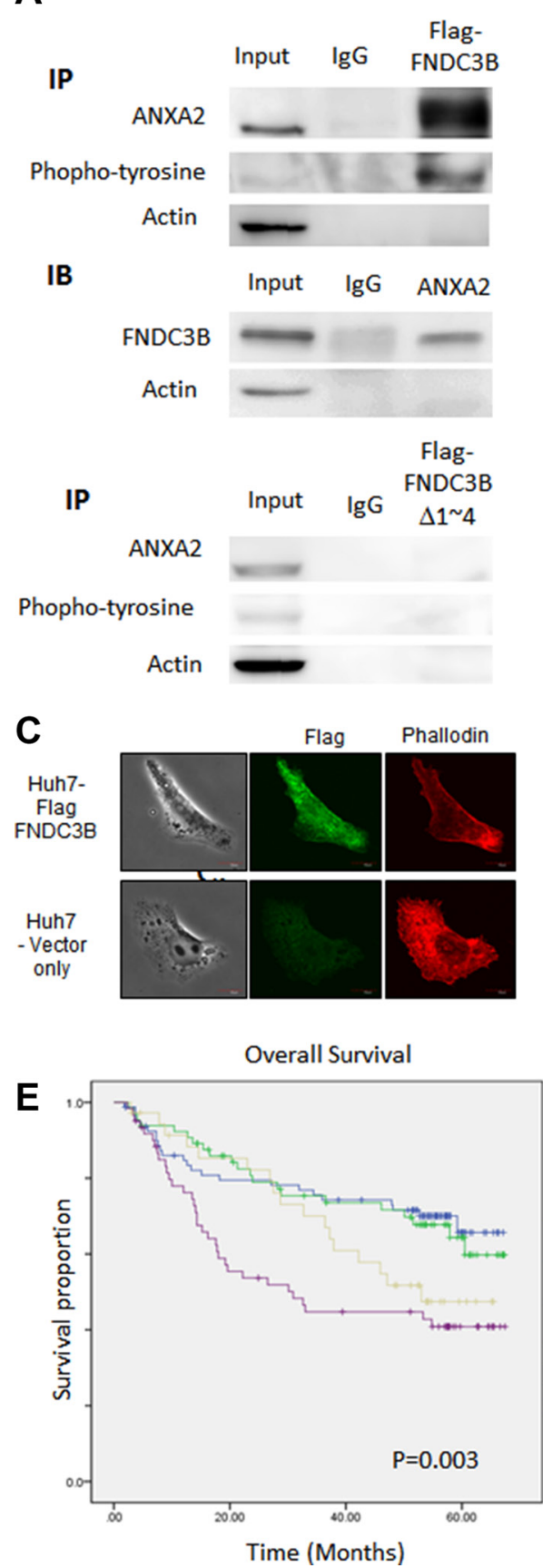

B

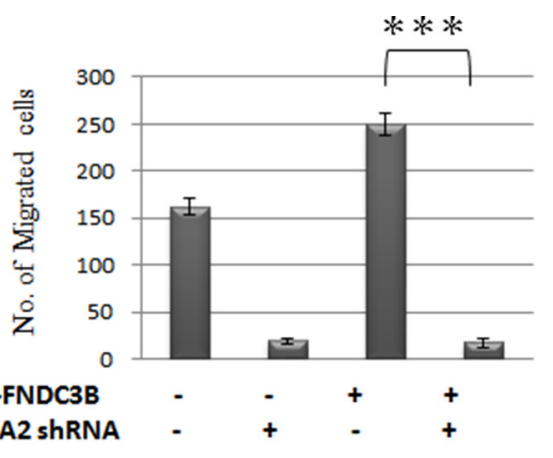

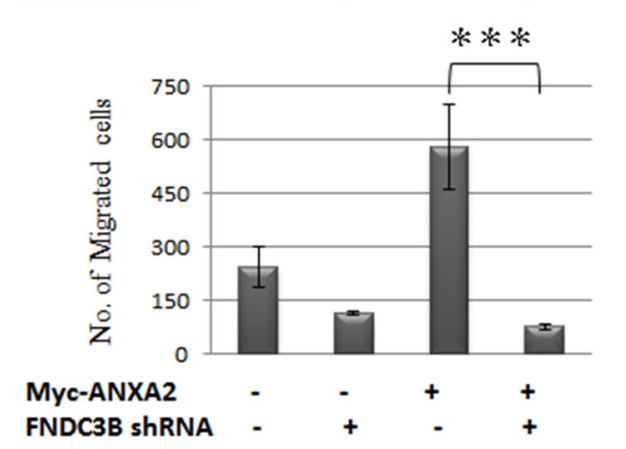

D

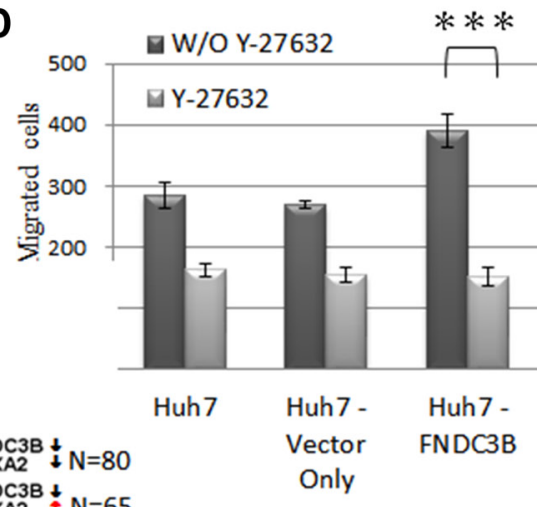

FNDC3B $\downarrow$ 政 $=65$

FNDC3B $+\mathrm{N}=36$

ANXA2

ANXA2 $\uparrow \mathrm{N}=61$

Figure 5: ANAX2 interacting FNIII domains 1-4 of FNDC3B were essential for FNDC3B enhanced cell migration. (A) Co-immunoprecipitation of Flag-FNDC3B and ANAX2, using lysates from Huh7 cells transfected with Flag-FNDC3B for immunoprecipitation (IP) with anti-Flag. (B) Migration assay for FNDC3B overexpressed Huh7 cells treated with $A N X A 2$ shRNA and $A N X A 2$ overexpressed Huh7 cells treated with FNDC3B shRNA. (C) Vector only and FNDC3B overexpression Huh7 cells observed by confocal microscopy. F-actin and Flag-FNDC3B were visualized with TRITC-conjugated phalloidin (red) and anti-Flag antibody (green), respectively. (D) Migration assay for $F N D C 3 B$ overexpressed Huh7 cells treated with $1 \mathrm{mM} / \mathrm{mL} \mathrm{Y27632.} \mathrm{***represents} p<0.005$. (E) Overall survival curves of HCC patients categorized according to FNDC3B and ANXA2 expression. $\uparrow$ and $\downarrow$ stand for high ( T/N $\geq 1.5)$ and low $(\mathrm{T} / \mathrm{N}<1.5)$ expression groups, respectively. 
Finally, disease-free survival analysis of cancer patients indicated that overexpression of FNDC3B correlates with recurrence and metastasis.

Tumor metastasis occurs in $90 \%$ of all cancer related deaths [37]. Thus, studies aimed at understanding metastasis might find therapeutic applications to improve patient survival. Our findings here suggest that FNDC3B could serve as diagnostic marker and therapeutic target for HCC.

\section{MATERIALS AND METHODS}

\section{Antibodies and cell culture}

Polyclonal anti-FNDC3B and monoclonal antiFlag M2 antibodies were purchased from Sigma. Monoclonal anti-ANXA2 antibody, Alexa Fluor-488 goat anti-mouse IgG and Alexa Fluor-488 goat antirabbit IgG were purchased from Invitrogen (Invitrogen, CA, USA). Monoclonal anti-beta-tubulin and polyclonal anti-GAPDH antibodies were purchased from Enogene (Enogene, Taiwan). Monoclonal anti-Myc (9E10) and monoclonal anti-phophotyrosine (4G10), were purchased from Millipore (Millipore Corporation, MA, USA). The polyclonal anti-calnexin antibody was purchased from GeneTex (GeneTex, CA, USA). HCC cell lines (HepG2, HuH7 and Mahlavu) were cultured in Dulbecco's modified Eagle's medium supplemented with $10 \%$ fetal bovine serum, $1 \%$ nonessential amino acids, and 1\% penicillin/ streptomycin (Invitrogen, Carlsbad, CA). Cells viability was determined by the cell proliferation assay every 24 hours for 4 days using the AlamarBlue reagent (AbD Serotec, UK).

\section{Small interfering RNA and lentiviral infection}

Short hairpin RNAs (shRNAs) targeting FNDC3B and $A N X A 2$ in the RNAi Consortium shRNA library were ordered from the National RNAi Core Facility (Academia Sinica, Taiwan). We selected and mixed the 2 or more effective shRNAs to knock down the expression of FNDC3B (TRCN0000082774 and TRCN0000082776) and ANXA2 (TRCN0000056144, TRCN0000056145, TRCN0000289381, and TRCN0000296322) by infection with 293T-produced lentivirus. For lentivirus production, the supernatant of the 293T culture was harvested at 24, 48 and $72 \mathrm{~h}$ after transfection with shRNA vectors. Targeted cells were then incubated with lentiviruses for $24 \mathrm{~h}$ with 8 $\mu \mathrm{g} / \mathrm{mL}$ polybrene (Sigma-Aldrich, MO, USA).

\section{Cell migration and invasion assays}

The migration assay was performed in 24-well transwell units with an $8 \mathrm{~mm}$ pore size polycarbonate membrane (BD Biosciences, CA, USA). Cells that were re-suspended in a serum-free medium were seeded into the upper chamber of the insert and then placed into the bottom chamber containing $10 \%$ fetal bovine serum as a chemoattractant. Cells were allowed to migrate for $24 \mathrm{~h}$, followed by methanol fixation and Giemsa staining (MERCK, Germany). Cells that did not migrate to the apical side of membrane were removed with a cotton swab. Migrated cells were photographed with a phasecontrast microscope. For the invasion assay, the membrane filters were coated with collagen to form a continuous thin layer. All experiments were performed in triplicates. Independent Student's $t$ tests were used to compare the continuous variables between the 2 groups.

\section{In vivo tumor metastasis}

For tail vein injections, cells $\left(5 \times 10^{6} /\right.$ mouse $)$ were intravenously injected into athymic BALB/c nude $(\mathrm{nu} / \mathrm{nu})$ mice at 4 weeks of age. The mice were sacrificed 8 weeks after injection. The livers and lungs from the sacrificed mice were harvested and fixed in $10 \%$ formalin followed by $75 \%$ ethanol. The number of metastatic tumors was then assessed. For orthotopical injections, cells $\left(5 \times 10^{6} /\right.$ mouse) were mixed with $50 \mu \mathrm{l}$ matrigel and injected into the left lobes of the livers of the athymic BALB/c nude $(\mathrm{nu} / \mathrm{nu})$ mice at 4 weeks of age. The animals were sacrificed 6 weeks after injection and the number of metastatic tumors was assessed.

\section{Immunohistochemistry and scoring}

Tissue microarrays (CS3 and CSN3) were purchased from SuperBioChips Labs (SuperBioChips Labs, Korea). The immunohistochemical methodology followed the standard protocol provided by the manufacturer's instructions. Immunohistochemical staining was scored according to the intensity of staining (no staining $=0$, weak staining $=1$, moderate staining $=2$, strong staining $=3$ ) and the extent of the cell stained $(0 \%=0,1-10 \%=1,11-50 \%$ $=2,>50 \%=3$ ). The final immunoreactive score was determined by adding the intensity scores with the extent of positivity scores of stained cells. Immunohistochemical analyses and scoring were performed by 3 independent investigators.

\section{Microarray analysis and kaplan-meier survival analysis}

For analysis of $F N D C 3 B$ expression in $\mathrm{HCCs}$, gene expression microarray datasets were downloaded from the public domain GEO database (GSE14520). The gene expression profiling experiments were performed using Affymetrix HG U133 series arrays. Since the scanned images of each array may have different overall brightness, the dChip (www.dchip.org) software was applied to normalize the brightness of the arrays to comparable levels. The tumor and comparative normal 
tissue arrays were entered into the dChip. The array corresponding to the median overall intensity was used as the baseline array to adjust the overall probe intensity level for all other arrays. Then the intensity of $F N D C 3 B$ normalized probe sets were output for analysis. $F N D C 3 B$ upregulation was defined as greater than 1.5 fold increase above the average intensity of 7 normal livers. KaplanMeier survival analysis was conducted using SPSS v.20 (IBM, USA).

\section{In-gel digestion and mass spectrometric analysis}

Distinct protein bands were manually picked from the SDS-PAGE and unstained in a solution of $25 \mathrm{mM}$ $\mathrm{NH}_{4} \mathrm{HCO}_{3}$ and $50 \%(\mathrm{v} / \mathrm{v})$ acetonitrile $(1: 1)$. The gel pieces were dehydrated in acetonitrile for $10 \mathrm{~min}$, vacuum dried, and rehydrated in $2 \%(\mathrm{v} / \mathrm{v}) \beta$-mercapteoethanol for $20 \mathrm{~min}$, followed by alkylation in $25 \mathrm{mM}$ ammonium bicarbonate containing 5\% 4-vinylpyridine, 50\% acetonitrile for $20 \mathrm{~min}$. Then gel pieces were washed twice with ammonium bicarbonate $(25 \mathrm{mM})$ at room temperature. Subsequently, the gels were vacuum dried, and finally digested with $25 \mathrm{ng}$ trypsin (Promega, WI, USA) in $25 \mathrm{mM}$ ammonium bicarbonate $\left(\mathrm{pH} \mathrm{8.5)}\right.$ at $37^{\circ} \mathrm{C}$ overnight. The tryptic peptides were extracted from gels and dried by Speed-Vac (Thermo Electron, MA, USA). Each tryptic digest was dissolved in $0.1 \%$ formic acid and loaded into an LTQ-Orbitrap Discovery hybrid mass spectrometer with a nanoelectrospray ionization source (ThermoElcetron, CA, USA) coupled to a nano-flow HPLC (Agilent Technologies 1200 series). Mobile phase solvent $\mathrm{A}$ and $\mathrm{B}$ were prepared as $0.1 \%$ formic acid in water and $0.1 \%$ formic acid in acetonitrile. Peptides were separated in a tip column $(13.5 \mathrm{~cm}$ length, $75 \mu \mathrm{m}$ inner diameter, $5 \mu \mathrm{m}$ beads, YMC-Gel, Lipid Chromatography) with a linear gradient of 3\%-40\% B for $90 \mathrm{~min}, 40 \%-95 \%$ $\mathrm{B}$ for $2 \mathrm{~min}, 95 \% \mathrm{~B}$ for $10 \mathrm{~min}$ at a flow rate of $0.5 \mu \mathrm{l} /$ min. Eluted peptides were ionized by a spray voltage of $2.3 \mathrm{kV}$ and introduced into the mass spectrometer. Mass spectrometric data were obtained using a data-dependent acquisition method, in which one full MS survey scan (m/z: 200-2000) was set at a high resolution of 30,000 (full-width at half-maximum) followed by scanning for the first six most highly charged ions $(2+$ and $3+)$. Fragmented peptide ions of each selected precursor peptide ion were generated by collision-induced dissociation (CID) using helium gas with $35 \%$ collision energy.

\section{Protein database search}

The TurboSequest search server (ver. 27, rev. 11; Thermo Electron, Waltham, MA, USA) was used to identify the peptide sequences against a UniProt human protein database (containing 127,738 protein sequences; released on February, 2015; http://www.uniprot.org/). A protein was identified when more than two peptides with an Xcorr score higher than 2.5 (for doubly charged ions) or 3.75 (for triply charged ions) were matched.

\section{Abbreviations}

HCC, hepatocellular carcinoma; FNDC3B, fibronectin type III domain containing 3B; ANXA2, annexin A2; FNIII, fibronectin type III domain; LC-MS/ MS, Liquid Chromatograph Tandem Mass Spectrometer.

\section{ACKNOWLEDGMENTS}

The authors acknowledge the technical services provided by High-throughput Genome Analysis Core Facility of the National Yang-Ming University VGH Genome Research Center (VYMGC). The Core Facility is supported by National Core Facility Program for Biotechnology (NCFPB), Ministry of Science and Technology

\section{CONFLICTS OF INTEREST}

No conflicts of interest to disclose.

\section{FUNDING}

Ministry of Science and Technology of Taiwan grant numbers NSC101-2320-B-010-066-MY3 and MOST 104-2320- B-010-028.

\section{REFERENCES}

1. Yang JD, Roberts LR. Hepatocellular carcinoma: A global view. Nat Rev Gastroenterol Hepatol. 2010; 7: 448-458.

2. El-Serag HB. Hepatocellular carcinoma. N Engl J Med. 2011; 365:1118-1127.

3. Poon RTP, Fan ST, Wong J. Risk factors, prevention, and management of postoperative recurrence after resection of hepatocellular carcinoma. Ann Surg. 2000; 232:10-24.

4. Zimmerman MA, Ghobrial RM, Tong MJ, Hiatt JR, Cameron AM, Hong J, Busuttil RW. Recurrence of hepatocellular carcinoma following liver transplantation: a review of preoperative and postoperative prognostic indicators. Ann Surg. 2008; 143:182-188; discussion 188.

5. Uchino K, Tateishi R, Shiina S, Kanda M, Masuzaki R, Kondo Y, Goto T, Omata M, Yoshida H, Koike K. Hepatocellular carcinoma with extrahepatic metastasis: clinical features and prognostic factors. Cancer. 2011; 117:4475-4483.

6. Senthilnathan S, Memon K, Lewandowski RJ, Kulik L, Mulcahy MF, Riaz A, Miller FH, Yaghmai V, Nikolaidis P, Wang E, Baker $\mathrm{T}$, Abecassis $\mathrm{M}$, Benson $\mathrm{AB}$, et al. Extrahepatic metastases occur in a minority of hepatocellular carcinoma patients treated with locoregional therapies: 
analyzing patterns of progression in 285 patients. Hepatology. 2012; 55:1432-1442.

7. Chen CF, Hsu EC, Lin KT, Tu PH, Chang HW, Lin $\mathrm{CH}$, Chen YJ, Gu DL, Wu JY, Chen YT, Hsu MT, Jou YS. Overlapping high-resolution copy number alterations in cancer genomes identified putative cancer genes in hepatocellular carcinoma. Hepatology. 2010; 52:1690-1701.

8. Tominaga $\mathrm{K}$, Kondo C, Johmura Y, Nishizuka M, Imagawa $M$. The novel gene fad104, containing a fibronectin type III domain, has a significant role in adipogenesis. FEBS Lett. 2004; 577:49-54.

9. Nishizuka M, Kishimoto K, Kato A, Ikawa M, Okabe M, Sato R, Niida H, Nakanishi M, Osada S, Imagawa M. Disruption of the novel gene fad104 causes rapid postnatal death and attenuation of cell proliferation, adhesion, spreading and migration. Exp Cell Res. 2009; 315:809-819.

10. Kishimoto K, Kato A, Osada S, Nishizuka M, Imagawa M. Fad104, a positive regulator of adipogenesis, negatively regulates osteoblast differentiation. Biochem Biophys Res Commun. 2010; 397:187-191.

11. Lin F, Ren XD, Pan Z, Macri L, Zong WX, Tonnesen MG, Rafailovich M, Bar-Sagi D, Clark RA. Fibronectin Growth Factor-Binding Domains Are Required for Fibroblast Survival. J Invest Dermatol. 2011; 131:84-98.

12. Obara M, Sakuma T, Fujikawa K. The third type III module of human fibronectin mediates cell adhesion and migration. J Biochem. 2010; 147:327-335.

13. van Kilsdonk JW, van Kempen LC, van Muijen GN, Ruiter DJ, Swart GW. Soluble adhesion molecules in human cancers: sources and fates. Eur J Cell Biol. 2010; 89:415-427.

14. Koide A, Bailey CW, Huang X, Koide S. The fibronectin type III domain as a scaffold for novel binding proteins. J Mol Biol. 1998; 284:1141-1151.

15. Bloom L, Calabro V. FN3: a new protein scaffold reaches the clinic. Drug Discov Today. 2009; 14:949-955.

16. Ji NY, Park MY, Kang YH, Lee CI, Kim DG, Yeom YI, Jang YJ, Myung PK, Kim JW, Lee HG, Kim JW, Lee K, Song EY. Evaluation of annexin II as a potential serum marker for hepatocellular carcinoma using a developed sandwich ELISA method. Int J Mol Med medicine. 2009; 24:765-771.

17. Yoon SY, Kim JM, Oh JH, Jeon YJ, Lee DS, Kim JH, Choi JY, Ahn BM, Kim S, Yoo HS, Kim YS, Kim NS. Gene expression profiling of human $\mathrm{HBV}$ - and/or HCV-associated hepatocellular carcinoma cells using expressed sequence tags. Int J Oncol. 2006; 29:315-327.

18. Mohammad HS, Kurokohchi K, Yoneyama H, Tokuda M, Morishita A, Jian G, Shi L, Murota M, Tani J, Kato K, Miyoshi H, Deguchi A, Himoto T, et al. Annexin A2 expression and phosphorylation are up-regulated in hepatocellular carcinoma. Int J Oncol. 2008; 33:1157-1163.

19. Zhao P, Zhang W, Tang J, Ma XK, Dai JY, Li Y, Jiang JL, Zhang SH, Chen ZN. Annexin II promotes invasion and migration of human hepatocellular carcinoma cells in vitro via its interaction with HAb18G/CD147. Cancer Sci. 2010; 101:387-395.

20. Rescher U, Ludwig C, Konietzko V, Kharitonenkov A, Gerke V. Tyrosine phosphorylation of annexin A2 regulates Rho-mediated actin rearrangement and cell adhesion. J Cell Sci. 2008; 121:2177-2185.

21. Zheng L, Foley K, Huang L, Leubner A, Mo G, Olino K, Edil BH, Mizuma M, Sharma R, Le DT, Anders RA, Illei PB, Van Eyk JE, et al. Tyrosine 23 phosphorylationdependent cell-surface localization of annexin A2 is required for invasion and metastases of pancreatic cancer. PLoS One. 2011; 6: e19390.

22. Garrido-Gomez T, Dominguez F, Quinonero A, Estella C, Vilella F, Pellicer A, Simon C. Annexin A2 is critical for embryo adhesiveness to the human endometrium by RhoA activation through F-actin regulation. FASEB J. 2012; $26: 3715-3727$.

23. Main AL, Harvey TS, Baron M, Boyd J, Campbell ID. The three-dimensional structure of the tenth type III module of fibronectin: an insight into RGD-mediated interactions. Cell. 1992; 71:671-678.

24. Grieve AG, Moss SE, Hayes MJ. Annexin A2 at the interface of actin and membrane dynamics: a focus on its roles in endocytosis and cell polarization. Int J Cell Biol. 2012; 2012:852430.

25. Flood EC, Hajjar KA. The annexin A2 system and vascular homeostasis. Vascul Pharmacol. 2011; 54:59-67.

26. Sharma MC, Sharma M. The role of annexin II in angiogenesis and tumor progression: a potential therapeutic target. Curr Pharm Des. 2007; 13:3568-3575.

27. Alfonso P, Canamero M, Fernandez-Carbonie F, Nunez A, Casal JI. Proteome analysis of membrane fractions in colorectal carcinomas by using 2D-DIGE saturation labeling. J Proteome Res. 2008; 7:4247-4255.

28. Deng S, Jing B, Xing T, Hou L, Yang Z. Overexpression of annexin A2 is associated with abnormal ubiquitination in breast cancer. Genomics Proteomics Bioinformatics. 2012; 10:153-157.

29. Longerich T, Haller MT, Mogler C, Aulmann S, Lohmann V, Schirmacher P, Brand K. Annexin A2 as a differential diagnostic marker of hepatocellular tumors. Pathol Res Pract. 2011; 207:8-14.

30. Yao H, Zhang Z, Xiao Z, Chen Y, Li C, Zhang P, Li M, Liu Y, Guan Y, Yu Y, Chen Z. Identification of metastasis associated proteins in human lung squamous carcinoma using two-dimensional difference gel electrophoresis and laser capture microdissection. Lung Cancer. 2009; 65:41-48.

31. Lu Y, Yi Y, Liu P, Wen W, James M, Wang D, You M. Common human cancer genes discovered by integrated gene-expression analysis. PLoS One. 2007; 2: e1149.

32. Zhang X, Liu S, Hu T, Liu S, He Y, Sun S. Up-regulated microRNA-143 transcribed by nuclear factor kappa B 
enhances hepatocarcinoma metastasis by repressing fibronectin expression. Hepatology. 2009; 50:490-499.

33. Cai C, Rajaram M, Zhou X, Liu Q, Marchica J, Li J, Powers RS. Activation of multiple cancer pathways and tumor maintenance function of the $3 \mathrm{q}$ amplified oncogene FNDC3B. Cell Cycle. 2012; 11:1773-1781.

34. Sawey ET, Chanrion M, Cai C, Wu G, Zhang J, Zender L, Zhao A, Busuttil RW, Yee H, Stein L, French DM, Finn RS, Lowe SW, et al. Identification of a therapeutic strategy targeting amplified FGF19 in liver cancer by Oncogenomic screening. Cancer Cell. 2011; 19:347-358.
35. Stangeland B, Mughal AA, Grieg Z, Sandberg CJ, Joel M, Nygard S, Meling T, Murrell W, Vik Mo EO, Langmoen IA. Combined expressional analysis, bioinformatics and targeted proteomics identify new potential therapeutic targets in glioblastoma stem cells. Oncotarget. 2015; 6:26192-26215. doi: 10.18632/oncotarget.4613.

36. Yang Y, Li D, Yang Y, Jiang G. An integrated analysis of the effects of microRNA and mRNA on esophageal squamous cell carcinoma. Mol Med Rep. 2015;12: 945-952.

37. Hanahan D, Weinberg RA. The hallmarks of cancer. Cell. 2000; 100:57-70. 